\title{
Evidence for integrating exercise training into the multidisciplinary management of non-specific chronic low back pain
}

Patrick J Owen, Clint T Miller, Niamh L Mundell, Paul Buntine, Daniel L Belavy

\section{Background \\ Worldwide, low back pain (LBP) is the leading cause of disability and affects $16 \%$ of the Australian population. Pain that lasts more than 12 weeks and is presumed lumbar musculoskeletal in origin is deemed non-specific chronic LBP. Managing LBP requires a multidisciplinary approach.}

\section{Objective}

The aim of this article is to provide evidence for incorporating exercise training into the multidisciplinary treatment plans of patients with non-specific chronic LBP.

\section{Discussion}

There is mounting evidence in support of the integration of exercise training for the multidisciplinary treatment of non-specific chronic LBP. Clinically meaningful reductions in pain intensity may be achieved with Pilates, aerobic, stabilisation/motor control and resistance exercise training. Clinically meaningful reductions in disability may be attained with resistance, stabilisation/motor control, water-based, Pilates and yoga exercise. Resistance and aerobic exercise can also improve mental health in this susceptible population group. The evidence suggests it is reasonable to consider including exercise-based services in the care team to enable patients with non-specific chronic LBP to transition to self-management.
LOW BACK PAIN (LBP) is the leading cause of disability ${ }^{1}$ and affects four million Australians ( $16 \%$ of the population) at any time. ${ }^{2}$ Pain lasting more than 12 weeks is deemed chronic LBP (CLBP). ${ }^{3}$ Of those who present with CLBP, approximately 90-95\% are classified as non-specific (presumed lumbar musculoskeletal origin, where further diagnostic imaging is unlikely to assist in diagnosis or management). ${ }^{3,4}$ Evidence-based clinical guidelines for managing non-specific LBP support using exercise training as a first-line therapy. ${ }^{5}$ Exercise training consists of physical activity that is planned, structured and repetitive, with an overall aim to improve and/or maintain physical fitness, health and/or function. This review aims to provide evidence-based principles for incorporating exercise training into the multidisciplinary treatment plans of patients with non-specific CLBP.

\section{Exercise training}

\section{Who is this for?}

The use of exercise training to manage non-specific CLBP is supported by international evidence-based clinical guidelines, ${ }^{5}$ as well as those specific to Australia. ${ }^{6}$ There is burgeoning evidence that exercise training may be suitable for patients with non-severe radicular syndromes, ${ }^{4}$ yet this is beyond the scope of this review. Therefore, the remainder of this review will focus on the role of exercise training in patients with non-specific CLBP.

\section{What exactly is provided?}

A recent systematic review of exercise training for non-specific CLBP identified a smorgasbord of exercise modalities that have been examined to date. ${ }^{7}$ They include, but are not limited to, resistance, aerobic, stabilisation/motor control, Pilates, McKenzie and stretching. Resistance exercises are designed to improve the strength, power, endurance or size of skeletal muscles. Aerobic exercises are designed to improve the efficiency and capacity of the cardiorespiratory system and can include walking, cycling and jogging. Stabilisation/motor control exercises target specific trunk muscles to improve control and coordination of the spine and pelvis. Pilates uses core principles such as centring, concentration, control, precision, flow and breathing. McKenzie exercises rely on principles such as repeated passive spine movement and sustained positions performed in specific directions. Stretching exercises include muscle lengthening using passive, static, isometric, ballistic or proprioceptive neuromuscular facilitation techniques. When two or more of these exercise modalities are combined, this is considered multimodal (eg a combined aerobic and resistance exercise training program).

\section{Evidence base}

In line with a shift towards the biopsychosocial model of managing non-specific CLBP, ${ }^{8}$ it is relevant to consider a range of benefits that may 
stem from exercise training. Common outcomes beyond reducing pain intensity may include improvements in disability (subjective physical function) and mental health. The efficacy and optimal mode of exercise training may vary depending on the outcome of interest.

Evidence (level 1; meta-analysis of homogenous trials) supports the use of aerobic, Pilates, resistance and stabilisation/motor control exercise training for reducing pain intensity. ${ }^{7}$ When considering a 100-point visual analogue scale, this corresponds with clinically meaningful reductions (ie more than 20 points) ${ }^{9}$ for Pilates ( -37 points), aerobic (-28 points), stabilisation/motor control ( -26 points) and resistance (-23 points) exercises (Figure 1). Given the range of exercise modalities capable of reducing pain intensity to a clinically meaningful degree, it appears that no one kind of exercise mode is superior; rather, active therapies, where a patient is encouraged to exercise in a progressive fashion, is optimal.

For reducing disability (improving subjective physical function), evidence (level 1) supports the use of aerobic, water-based, multimodal, Pilates, resistance, stabilisation/motor control and yoga exercise training. ${ }^{7}$ When converted to units on the Oswestry Disability Index, a commonly used tool for subjective disability in patients with non-specific CLBP, a clinically meaningful reduction (-11 points) was observed for resistance (-14 points), stabilisation/motor control (-14 points), water-based (-12 points), yoga ( -11 points) and Pilates ( -11 points) exercises (Figure 2). Given that Pilates, resistance and stabilisation/motor control exercises are capable of reducing both pain intensity and disability to a clinically meaningful degree, these exercise modalities appear particularly promising in managing non-specific CLBP.

Table 1 gives an example of a six-month resistance exercise-based program ${ }^{10}$ shown to improve pain intensity and disability in patients with non-specific CLBP.

Evidence (level 1) also supports using aerobic and resistance exercise training to improve mental health. ${ }^{7}$ Notably, fewer studies $(n=16)$ have been published using these outcome measures, and vast heterogeneity exists in outcomes used to quantify mental health. The emergence of resistance exercise training as optimal for mental health further adds to the contention that this modality should be specifically considered, given the range of benefits in patients with non-specific CLBP.

Other outcomes and modes of exercise training have received comparatively less attention in patients with non-specific CLBP, yet evidence continues to mount.
A meta-analysis of seven studies showed that exercise training reduced fear-avoidance behaviours; ${ }^{11}$ another meta-analysis of five studies showed that horse riding simulator exercise training reduced pain intensity, yet concluded evidence was lacking for real horse riding (equine-assisted therapy). ${ }^{12}$ Additionally, evidence from a recent randomised clinical trial showed that while beneficial for reducing pain intensity, disability and fear avoidance, ${ }^{13}$ a six-month general

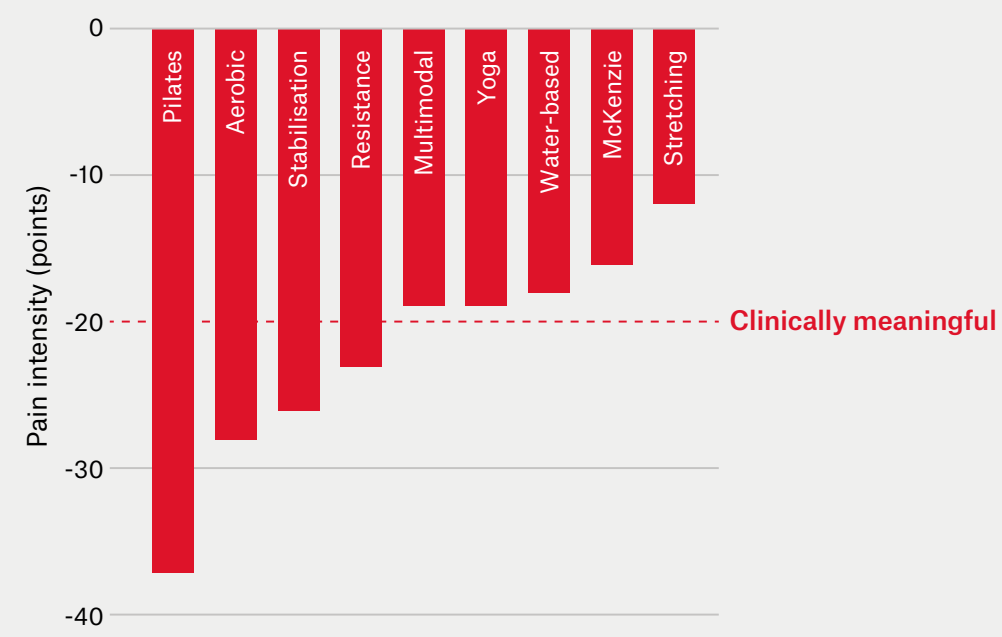

Figure 1. Effects of exercise training modes on pain intensity

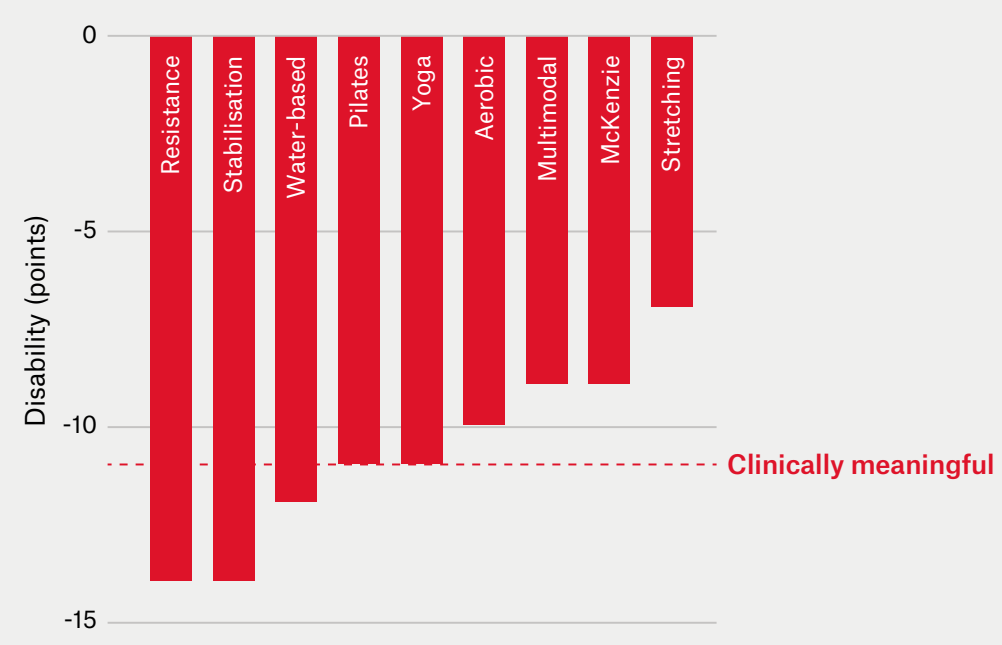

Figure 2. Effects of exercise training modes on disability 


\section{Table 1. An example of a full-body resistance exercise-based program for patients with non-specific chronic low back pain ${ }^{10}$}

Weeks Goal: familiarisation, motor control and local muscular endurance

1-4 Intensity: 12-15 reps at two reps below volitional fatigue $\times$ two sets, one-minute rest between sets

Frequency: two sessions per week

Time under tension: two seconds concentric, two seconds eccentric

Progression: after two sets $\times 15$ reps at two consecutive training sessions, resistance is increased

\section{Weeks Goal: muscular strength}

5-10 Intensity: 6-10 reps at two reps below volitional fatigue $\times 2-3$ sets, two-minute rest between sets

Frequency: two sessions per week

Time under tension: two seconds concentric, two seconds eccentric

Progression: after two sets $\times 10$ reps at two consecutive training sessions, workload increases to three sets, then progression is made through increased resistance

Week 11 Goal: de-load

Intensity: 10 reps at $80 \%$ of resistance in previous week $\times$ three sets, two-minute rest between sets

Frequency: two sessions per week

Time under tension: two seconds concentric, two seconds eccentric Progression: not applicable

Weeks Goal: local muscular endurance

12-15 Intensity: 12-15 reps at two reps below volitional fatigue $\times$ three sets, one-minute rest between sets

Frequency: 1-2 sessions per week

Time under tension: five seconds concentric, five seconds eccentric

Progression: after three sets $\times 15$ reps at two consecutive training sessions, resistance is increased

\section{Weeks Goal: muscular strength}

16-19 Intensity: 6-10 reps at two reps below volitional fatigue $\times 3-4$ sets, two-minute rest between sets

Frequency: 1-2 sessions per week

Time under tension: two seconds concentric, two seconds eccentric

Progression: after three sets $\times 10$ reps at two consecutive training sessions, workload increases to four sets, then progression is made through increased resistance

Weeks Goal: local muscular endurance

20-25 Intensity: 20-25 reps at two reps below volitional fatigue $\times$ three sets, one-minute rest between sets

Frequency: 1-2 sessions per week

Time under tension: 1-2 seconds concentric, 1-2 seconds eccentric

Progression: after three sets $\times 25$ reps at two consecutive training sessions, resistance is increased

reps, repetitions

strength and conditioning exercise training program did not improve the health of degenerated intervertebral discs in a cohort of patients with non-specific CLBP. ${ }^{14}$

\section{Summary}

There is mounting evidence in support of the integration of exercise training for the multidisciplinary treatment of non-specific CLBP. However, high-quality estimates from the Australian general practice setting suggest that only 19\% of patients with non-specific CLBP are referred to exercise training. ${ }^{15}$ Therefore, general practitioners should consider including exercise-based services to complement the care team and assist transition to self-management for patients with non-specific CLBP. In particular, resistance exercise training appears to result in clinically meaningful reductions in pain intensity and disability, as well as improvements in mental health; this mode should be specifically considered when choosing the type of exercise training for managing non-specific CLBP.

\section{Authors}

Patrick J Owen BEx\&SportSc (Hons), PhD, Dean's Postdoctoral Research Fellow, Deakin University, Institute for Physical Activity and Nutrition, School of Exercise and Nutrition Sciences, Vic

Clint T Miller MAppSc (ExRehab), GCertHELT, PhD ESSAM, Lecturer in Clinical Exercise Physiology, Deakin University, Institute for Physical Activity and Nutrition (IPAN), School of Exercise and Nutrition Sciences, Vic; Accredited Exercise Physiologist, Deakin University, IPAN, School of Exercise and Nutrition Sciences, Vic

Niamh L Mundell MAppSc (ExRehab), GCertHELT, ESSAM, Lecturer in Clinical Exercise Physiology, Deakin University, Institute for Physical Activity and Nutrition (IPAN), School of Exercise and Nutrition Sciences, Vic; Accredited Exercise Physiologist, Deakin University, IPAN, School of Exercise and Nutrition Sciences, Vic

Paul Buntine MBBS, FACEM, Director of Emergency Medicine Research, Emergency Department, Box Hill Hospital - Eastern Health, Vic; Emergency Physician, Emergency Department, Box Hill Hospital - Eastern Health, Vic; Adjunct Senior Lecturer, Eastern Health Clinical School, Monash University, Vic

Daniel L Belavy BPhty, GCertHELT, PhD, Associate Professor of Exercise and Musculoskeletal Health, Deakin University, Institute for Physical Activity and Nutrition, School of Exercise and Nutrition Sciences, Vic

Competing interests: None.

Funding: This work was funded by Musculoskeletal Australia (formerly MOVE Muscle, Bone \& Joint Health; CONTR2017/00399).

Provenance and peer review: Not commissioned, externally peer reviewed.

Correspondence to: belavy@gmail.com

\section{Acknowledgements}

The authors thank their collaborators on the wider funded project: Simone JJM Verswijveren, Scott D Tagliaferri, Professor Helena Brisby and Associate Professor Steven J Bowe.

\section{References}

1. GBD 2015 Disease and Injury Incidence and Prevalence Collaborators. Global, regional, and national incidence, prevalence, and years lived 
with disability for 310 diseases and injuries, 1990-2015: A systematic analysis for the global burden of disease study 2015. Lancet 2016;388(10053):1545-602. doi: 10.1016/S01406736(16)31678-6.

2. Australian Institute of Health and Welfare. Back problems. Canberra, ACT: AlHW, 2020. Available at www.aihw.gov.au/reports/chronicmusculoskeletal-conditions/back-problems/ contents/what-are-back-problems [Accessed 18 December 2020].

3. Koes BW, van Tulder M, Lin CW, Macedo LG, McAuley J, Maher C. An updated overview of clinical guidelines for the management of non-specific low back pain in primary care. Eur Spine J 2010;19(12):2075-94. doi: 10.1007/ s00586-010-1502-y.

4. Bardin LD, King P, Maher CG. Diagnostic triage for low back pain: A practical approach for primary care. Med J Aust 2017;206(6):268-73. doi: 10.5694/mja16.00828.

5. Oliveira CB, Maher CG, Pinto RZ, et al. Clinical practice guidelines for the management of nonspecific low back pain in primary care: An updated overview. Eur Spine J 2018;27(11):2791-803. doi: 10.1007/s00586-018-5673-2.

6. NSW Agency for Clinical Innovation. Management of people with acute low back pain - Model of care. Chatswood, NSW: NSW Health, 2016.

7. Owen PJ, Miller CT, Mundell NL, et al. Which specific modes of exercise training are most effective for treating low back pain? Network meta-analysis. Br J Sports Med 2020;54(21):1279-87. doi: 10.1136/ bjsports-2019-100886.

8. Tagliaferri SD, Miller CT, Owen PJ, et al. Domains of chronic low back pain and assessing treatment effectiveness: A clinical perspective. Pain Pract 2020;20(2):211-25. doi: 10.1111/papr.12846.
9. Mannion AF, Balagué F, Pellisé F, Cedraschi $C$. Pain measurement in patients with low back pain. Nat Rev Rheumatol 2007;3(11):610-18. doi: 10.1038/ncprheum0646.

10. Simson KJ, Miller CT, Ford J, et al. Optimising conservative management of chronic low back pain: Study protocol for a randomised controlled trial. Trials 2017;18(1):184. doi: 10.1186/s13063-0171913-8.

11. Hanel J, Owen PJ, Held S, et al. Effects of exercise training on fear-avoidance in pain and pain-free populations: Systematic review and meta-analysis. Sports Med 2020;50(12):2193-207. doi: 10.1007/ s40279-020-01345-1.

12. Collado-Mateo D, Lavín-Pérez AM, Fuentes García JP, García-Gordillo MÁ, Villafaina S. Effects of equine-assisted therapies or horse-riding simulators on chronic pain: A systematic review and meta-analysis. Medicina (Kaunas) 2020;56(9):444. doi: 10.3390/ medicina56090444.

13. Tagliaferri SD, Miller CT, Ford JJ, et al. Randomized trial of general strength and conditioning versus motor control and manual therapy for chronic low back pain on physical and self-report outcomes. J Clin Med 2020;9(6):1726. doi: 10.3390/ jcm9061726.

14. Owen PJ, Miller CT, Rantalainen T, et al. Exercise for the intervertebral disc: A 6-month randomised controlled trial in chronic low back pain. Eur Spine J 2020;29(8):1887-99. doi: 10.1007/s00586-02006379-7.

15. Kamper SJ, Logan G, Copsey B, et al. What is usual care for low back pain? A systematic review of health care provided to patients with low back pain in family practice and emergency departments. Pain 2020;161(4):694-702. doi: 10.1097/j.pain.0000000000001751. 